This document is confidential and is proprietary to the American Chemical Society and its authors. Do not copy or disclose without written permission. If you have received this item in error, notify the sender and delete all copies.

\title{
The Secret Life of Collagen: Temporal Changes in Nanoscale Fibrillar Pre-Strain and Molecular Organization During Physiological Loading of Cartilage
}

\begin{tabular}{|r|l|}
\hline Journal: & ACS Nano \\
\hline Manuscript ID & nn-2017-005633.R4 \\
\hline Manuscript Type: & Article \\
\hline Complete List of Authors: & $\begin{array}{l}\text { Inamdar, Sheetal; Queen Mary, University of London, School of } \\
\text { Engineering and Materials Science } \\
\text { Knight, David; Oxford Biomaterials Ltd, Units 14-15, Galaxy House, New } \\
\text { Greenham Business Park, Newbury RG19 6HW UK } \\
\text { Terrill, Nicholas; Diamond Light Source, } \\
\text { Karunaratne, Angelo; Imperial College London, Department of } \\
\text { Bioengineering } \\
\text { Cacho-Nerin, Fernando; Graz University of Technology, Institute of } \\
\text { Inorganic Chemistry } \\
\text { Knight, Martin; University of London, Mile End Road, IRC in Biomedical } \\
\text { Materials,Queen Mary } \\
\text { Gupta, Himadri; Queen Mary, University of London, School of Engineering } \\
\text { and Materials Science }\end{array}$ \\
\hline
\end{tabular}




\title{
The Secret Life of Collagen: Temporal Changes
}

\author{
in Nanoscale Fibrillar Pre-Strain and Molecular
}

\section{Organization During Physiological Loading of}

\section{Cartilage}

Sheetal R. Inamdar ${ }^{1}$, David P. Knight ${ }^{2}$, Nicholas J. Terrill ${ }^{3}$, Angelo Karunaratne ${ }^{4, t}$, Fernando Cacho-Nerin ${ }^{3}$, Martin M. Knight ${ }^{1}$ and Himadri S. Gupta ${ }^{1 *}$

1. Institute of Bioengineering, School of Engineering and Materials Science, Queen Mary University of London, London

2. Bridge Cottage, Cwmyoy, Abergavenny, Wales

3. Diamond Light Source, Harwell Science and Innovation Campus, Harwell, Didcot

4. Department of Bioengineering, Imperial College London, London 


\begin{abstract}
Articular cartilage is a natural biomaterial whose structure at the micro- and nanoscale is critical for healthy joint function and where degeneration is associated with widespread disorders such as osteoarthritis. At the nanoscale, cartilage mechanical functionality is dependent on the collagen fibrils and hydrated proteoglycans that form the extracellular matrix. The dynamic response of these ultrastructural building blocks at the nanoscale, however, remains unclear. Here we measure time-resolved changes in collagen fibril strain, using small angle X-ray diffraction during compression of bovine and human cartilage explants. We demonstrate the existence of a collagen fibril tensile pre-strain, estimated from the D-period at approximately $1-2 \%$, due to osmotic swelling pressure from the proteoglycan. We reveal a rapid reduction and recovery of this prestrain which occurs during stress relaxation, approximately 60 seconds after the onset of peak load. Furthermore, we show that this reduction in pre-strain is linked to disordering in the intrafibrillar molecular packing, alongside changes in the axial overlapping of tropocollagen molecules within the fibril. Tissue degradation in the form of selective proteoglycan removal disrupts both the collagen fibril pre-strain and the transient response during stress relaxation. This study bridges a fundamental gap in the knowledge describing time-dependent changes in collagen pre-strain and molecular organisation that occur during physiological loading of articular cartilage. The ultrastructural details of this transient response is likely to transform our understanding of the role of collagen fibril nano-mechanics in the biomechanics of cartilage and other hydrated soft tissues.
\end{abstract}


Keywords: collagen fibrils; proteoglycans; cartilage; nanoscale mechanics; in situ x-ray nanomechanics; synchrotron microbeam $\mathrm{x}$-ray diffraction; prestressed fibrils

The existence of pre-tensed, extensible fibrils in a hydrated, amorphous gel is a widespread characteristic of both natural and synthetic nanostructured soft matter systems. ${ }^{1-5}$ The mechanical functions of these composites are dependent on the interactions between the fibril phase, the surrounding gel phase and free- and bound water. However, the critical nanoscale mechanics, in particular the extension, reorientation and strain of the nanofibrous phase, are challenging to determine experimentally. Articular cartilage $(A C)$ is a biological example where such a nanofibrous architecture is believed to be essential in providing the tissue with its mechanical functionality. In particular, the main function of articular cartilage is to act as a low friction, load bearing material with the ability to deform under dynamic compressive loading, thereby reducing stress to the underlying bone. ${ }^{8-10}$ The degradation of articular cartilage is associated with the musculoskeletal disorder osteoarthritis (OA), affecting $\sim 12-15 \%$ of the population between $25-74$ years of age. ${ }^{6,7}$ The tissue structure, which provides the ability to withstand both high and repetitive loading, exhibits a depth dependent anisotropy in terms of the content and distribution of the constituent macromolecules. Collagen fibrils play a crucial role in the mechanical functionality of articular cartilage, through interactions with the other main extracellular matrix (ECM) component, hydrated proteoglycans. ${ }^{7,11,12}$ The $3 \mathrm{D}$ orientations of these fibrils vary with depth such that they are parallel to the surface in the superficial zone, randomly orientated in the transitional zone and perpendicular to the surface in the deep zone adjacent to the subchondral bone (Fig. 1C). The negatively charged entangled 
proteoglycans create an osmotic swelling pressure which is resisted by the collagen fibrils in order to maintain equilibrium. ${ }^{13}$ This mechanism requires the collagen fibres to be permanently under a state of tensile pre-strain. ${ }^{14,15}$ The magnitude of this pre-strain, however, has never been quantified experimentally. Indeed, little is known about the collagen fibril nano-mechanics in situ within the cartilage extracellular matrix.

While many investigations have used ultrastructural and microscopic imaging methods to help to understand collagen structure-function relations in cartilage, these studies have largely ignored temporal changes during loading and unloading and do not provide quantitative information on the mechanics at the fibrillar level. These investigations primarily include electron microscopy and vibrational spectroscopy to image collagen fibril orientation and content in static conditions, alongside estimates of increasing fibre diameter with depth from the articular surface. ${ }^{16-18}$ The present study uses time-resolved synchrotron small angle X-ray diffraction (SAXD) combined with in situ mechanics to directly quantify the kinetic response of collagen fibrils in cartilage. As described in prior work on tendon, ${ }^{19,20}$ bone ${ }^{21-23}$ and $\operatorname{skin}^{24}$ the periodic electron density profile in the collagen fibrils, with an axial repeat length $D$ of $\sim 65-67 \mathrm{~nm}$, leads to diffraction peaks in the low wavevector (small-angle) range. Shifts in the diffraction peaks are markers of fibril-level strain, and the orientation and shape of the peaks are markers of fibril direction and dispersion. X-ray diffraction has previously been used to determine cartilage collagen fibril orientation as a function of depth below the articular surface. ${ }^{25}$ Using SAXD, Moger and coworkers ${ }^{27}$ found that fibrils in regions of the tissue with relatively low loading possessed a lower degree of orientation and the converse was true for the higher loaded regions. Furthermore they used the technique to show 
that in early lesions the fibrillar orientation was disrupted. The same group also later showed that with applied stress of $4.8-6.0 \mathrm{MPa}$ there was a 10 degree rotation of the collagen fibrils near the tide mark and at greater stresses, fibrils began to "crimp" ${ }^{26,27}$ Here we use SAXD to measure the collagen fibril strain and the temporal dynamics during stress relaxation using a microfocus X-ray beam to spatially resolve fibril ultrastructure in the deep and transitional zones separately. We investigate collagen nanomechanics in healthy bovine and human cartilage explants and how this behaviour is affected by removal of the hydrated proteoglycans by enzymatic digestion. Such fundamental collagen nano-mechanics behaviour in cartilage will provide insight into its mechanical behaviour and functionality.

\section{Results and Discussion:}

\section{Zonal variation in collagen fibre orientation}

Using SAXD in conjunction with a specially built mechanical testing system (adapted from Karunaratne et al. ${ }^{21}$ ) (Fig. 1a) we have produced diffraction data which we have used to quantify the collagen orientation and D-period in unstrained and compressed articular cartilage, as shown schematically in Fig. 1b. Our SAXD patterns show that the collagen fibres are parallel to the articular surface in the superficial zone and perpendicular to the surface in the deep zone, while they have a more isotropic distribution in the transitional zone (Fig. 1c) confirming the well-established spatial pattern of collagen orientation in articular cartilage..$^{9,27,28}$

\section{Sudden transient reduction in collagen fibril pre-strain during stress relaxation}


We reveal a time-dependent ultrastructural change observed in collagen fibrils during compression of articular cartilage. During the stress relaxation phase at constant applied tissue strain (Fig. 2a), there is a sudden transient reduction in collagen D-period (Fig. 2b). This indicates a reduction in the level of fibril tensile pre-strain. This behaviour occurs approximately $60-100 \mathrm{~s}$ after the peak stress and is followed by a rapid return to the original loaded D-period. The whole event lasts approximately 50-80 s and occurs without any perceptible fluctuation in the macroscopic stress relaxation response (Fig. $2 a$ inset). This transient reduction in pre-strain indicated by change in the collagen Dperiod was observed in both bovine (Fig. 2) and human articular cartilage (Fig. 3). There was no statistically significant difference between the transitional and deep zones in terms of the timing of the sudden reduction in D-period after the onset of the application of compression, with mean values ( \pm standard error of the mean or \pm SEM) of 60.0 $( \pm 11.4)$ and $82.5( \pm 11.1)$ seconds respectively (Fig. 3c). Immediately after compression of human articular cartilage, there was a slight reduction in D-period of approximately $0.5 \%$. This was statistically significant in the deep zone with a change in D-period from $65.6( \pm 0.07, n=4) n m$ to $65.3( \pm 0.11, n=4) n m($ mean \pm SEM, $p<0.05$, Fig. $3 d)$.

\section{Calculation of collagen pre-strain and the effect of swelling pressure}

To examine the mechanism regulating the observed transient changes in collagen pre-strain and $D$ period, we next investigated the effect of proteoglycan swelling pressure. For these studies, bovine articular cartilage explants were examined with and without pre-treatment with chondroitinase ABC (Sigma-Aldrich, Poole, UK). Treatment resulted in loss of sulphated glycosaminoglycan chains (sGAG) into the media (Fig. S1) 
and changes in macroscopic tissue mechanics with a reduction in mean $( \pm S E M)$ tangent modulus from $5.9 \mathrm{MPa}( \pm 0.52)$ in the untreated group to $3.4 \mathrm{MPa}( \pm 0.37)$ in the $0.1 \mathrm{U} / \mathrm{ml}$ treatment group $(\mathrm{p}<0.001 ;$ Fig. $\mathrm{S} 2$ and $\mathrm{S} 3$ ). In the untreated bovine cartilage samples, compression induced the sudden transient reduction in collagen D-period in both transitional and deep zones (Fig. 4a,b) as seen in human cartilage (Fig. 3) indicative of a reduction in fibril tensile strain. In the unloaded cartilage, enzymatic degradation of the proteoglycan produced a significant reduction in D-period in both the transitional (Fig. 4e) and deep zones (Fig. 4f). This reduction is accompanied by an increased scatter in the D-period variation for the chondroitinase-treated samples (Fig. $4 a$ and $4 \mathrm{~b}$, lower rows), especially in the transitional zone, suggesting mechanical disruption of the fibrillar network, as well as a reduction in pre-strain. These findings demonstrate the relationship between proteoglycan/water content and collagen fibrillar pre-strain. Specifically, the swelling pressure, exerted by the hydrated proteoglycan, causes tensile strain of the collagen fibres as predicted by established models of cartilage biomechanics. ${ }^{29-31}$ Without knowledge of the collagen D-period in the complete absence of any swelling pressure it is not possible to calculate the exact level of collagen pre-strain. However, assuming that the chondroitinase treatment removes all the intrinsic swelling pressure, the collagen fibres would experience a tensile pre-strain of approximately $1.5 \%$. Based on previous estimates of a single collagen fibril stiffness of $\sim 500 \mathrm{MPa},{ }^{32}$ this level of pre-strain is equivalent to a tensile force resisting the swelling pressure of $\sim 0.3 \mu \mathrm{N}$ / fibril for a fibril radius of $\sim 100 \mathrm{~nm}$ (corresponding to a stress of $\sim 7.5 \mathrm{MPa}$ ). Furthermore the results suggest that the sudden transient 
reduction in D-period is equivalent to a $60 \%$ reduction in total collagen pre-strain (Fig. 2).

Interestingly, in the transitional zone of bovine cartilage digested with chondroitinase to remove proteoglycan, compression resulted in a significant increase in D-period at peak load (Fig. 4e), in contrast to the lack of change seen in both zones of untreated control samples (Fig. 3d and 4e,f). Further, it is seen that the sudden decrease in collagen D-period is much less clear in the transitional zone of the enzymatically digested tissue, compared to controls in the same region (Fig. 4a) and both groups in the deep zone (Fig. 4b). It is possible that these effects are related to fibril-fibril interaction between zones. Horizontally oriented superficial zone collagen fibrils are continuously connected to the transitional zone fibrils via the well-known arcade-like collagen fibril structure. ${ }^{33,34}$ On vertical compression, the superficial zone fibrils (at $90^{\circ}$ to the loading direction) will extend in tension, and (due to their interconnection to the transitional zone) exert a tensile force on the transitional zone fibrils. We suggest that in native cartilage, fibrils are pre-strained by the proteoglycans almost to their maximum limit, while on enzymatic digestion, this pre-strain is lost. Therefore, in the chondroitinase digested tissue, the fibrils are more flexible due to the loss of pre-strain and have a greater range to extend (increase) in $D$ when tension is applied. We can speculate, therefore, that the tensile force from the superficial zone fibrils will not have any effect on the transitional zone fibrils in native cartilage (as the fibrils cannot extend further) but in chondroitinase-digested cartilage, leads to an increase in D-period for the transitional zone fibrils. 


\section{Intra-fibrillar reordering correlating to a reduction in D-period}

In order to understand the origins of the transient reduction in fibrillar pre-strain (in terms of the D-period), the structural mechanisms enabling mechanical homeostasis between fibrils and the hydrated proteoglycans first much be considered. The resultant loss of pre-strain is most likely due to the reduction of the localised proteoglycan-induced swelling pressure and will arise if the hydration of the proteoglycans is lowered. The osmotic and swelling pressures inside cartilage are determined by a combination of water content in both the proteoglycan and collagen components, as well as ionic strength and $\mathrm{pH}$ of the immersing medium, and it has been shown that ionic strength variations can alter intrafibrillar spacing. ${ }^{35}$ Here we have applied controlled compression, whereby some of the water associated with the proteoglycans will undergo stress induced movement, thus expelling water from the interstitial space within the tissue. This effect, and the associated reduction in the fibrillar pre-strain, will also lead to further structural changes within the fibrillar network.

Three hypothetical scenarios to accommodate such local changes are (i) intrafibrillar rearrangement and disordering, (ii) flow of water from the intrafibrillar space to the interfibrillar compartment, and (iii) fibrillar reorientation. Intrafibrillar rearrangement of tropocollagen molecules, specifically leading to changes in the gap to overlap ratio, have a characteristic and quantifiable effect on the Bragg peak intensities in the meridional SAXD pattern. If mechanism (i) is correct then we would expect either changes in the relative intensity ratios of different Bragg peaks in the SAXD pattern due to a change in the ratio of overlap zone in the fibril to the fibrillar D-stagger (O/D) ${ }^{36,37}$ 
and/or a disorder-induced reduction of peak intensities, with higher orders (larger wave vector) reducing more than lower orders, via a Debye-Waller term. ${ }^{36}$ In this scenario, assuming a step-function line-shape for the electron density variation across in the gap and overlap zones in the fibril, it is possible to derive analytical expressions for the ratios of the intensities of different orders of Bragg peaks (Equations (1)-(2), Materials and Methods), as functions of $\mathrm{D}$, the overlap/D-period ratio (O/D) and a Debye-Waller term ( $\kappa)$ proportional to the axial disorder at the gap/overlap interface ${ }^{36,37}$ Peak intensities are denoted as $I_{n}$ with $n$ the peak order (e.g. $I_{5}$ for the $5^{\text {th }}$ order peak), and are measured from the total area under each Bragg peak (Materials and Methods). For mechanism (ii), the lateral intermolecular spacing of the tropocollagen molecules would reduce from the known value for hydrated collagen fibrils $\left(d_{\mathrm{m}} \sim 1.5 \mathrm{~nm}\right)$ towards the value for dehydrated fibrils $\left(d_{\mathrm{m}} \sim 1.1 \mathrm{~nm}\right) \cdot{ }^{38,39}$ Lastly, for mechanism (iii), changes to predominant fibrillar orientation (from the SAXD I(X) position) would occur transiently at the point of minimum D. To obtain an initial indication as to which one of these mechanisms is predominant, we analyse in more detail the SAXD patterns in a representative example (sample shown in Fig. 2).

Fig. $5 A$ ) and $B$ ) show the variation of $I_{7} / I_{5}$ and $I_{8} / I_{5}$ with time, overlaid with a trace of the D-period variation to observe any correlated change. It is seen that near the minimum of $D$, the peak intensity ratios likewise exhibit a minimum. Viewing just the time-zone of the reduction of $D($ Fig. $5 C)$ ) it is observed that the ratios first decrease (zone 1), then vary in opposite directions, with $I_{8} / I_{5}$ increasing and $I_{7} / I_{5}$ decreasing, in zone 2 , which contains the minimum of $D$. In the subsequent zone 3 , there is a recovery of intensity 
ratios to near original values. From the disordered step-function model of the gap/overlap zone of the collagen fibril, a "phase-diagram" of intensity ratios for varying levels of disorder can be constructed, and is shown in Fig. 5D. By varying the disorder term $\kappa$ until a mutually consistent solution for $O / D$ is obtained $\left(\kappa \sim 1.75 \mathrm{~nm}^{2}\right.$; see Supplementary Information section S.2) for the initial values of $I_{7} / I_{5} \sim 0.25$ and $I_{8} / I_{5} \sim 0.15$, an initial value for $O / D \sim 0.466$ is found. This value is very close to previous $O / D$ values of $0.46-0.47$ reported in vertebrate tissues like tendon. ${ }^{40}$ The concurrent decrease in intensity in zone $1(\mathrm{Oa} \rightarrow \mathrm{Ob}$ in Fig. 5D; concurrently $\mathrm{Ea} \rightarrow \mathrm{Eb})$ can be modelled by a further increase in the disorder term $\kappa$ from $1.75 \mathrm{~nm}^{2}$ to $4.00 \mathrm{~nm}^{2}$. The next stage (zone $2 ; \mathrm{Ob} \rightarrow \mathrm{Oc}$ in Fig. 5D) can be reproduced by keeping $\kappa$ fixed and reducing O/D from 0.466 to 0.458 (leftward arrows Fig. $5 \mathrm{D}$ ), as $I_{7} / I_{5}$ and $I_{8} / I_{5}$ vary in opposite directions near $\mathrm{O} / \mathrm{D} \sim 0.466$. The last stage $\mathrm{Oc} \rightarrow \mathrm{Od}$, involving a combined reduction in disorder and increase in O/D, returns intensity ratios and $D$ to near initial values, as observed. $A$ reduction from the initial values exists $\left(\kappa=2.8 \mathrm{~nm}^{2}\right.$ at the end vs $1.75 \mathrm{~nm}^{2}$ at the start), indicating residual disorder. Line-plots of these variations in intensity ratios are shown, together with the experimental data from Fig. 5C, in Supplementary Fig. S4. Because of the high observed sensitivity of the intensity ratio changes to small variations in O/D and disorder, it is possible that across different samples, precise details of the timesequence of intensity ratio variations may differ somewhat.

These findings support the idea that change of intrafibrillar disorder and axial arrangement of tropocollagen molecules (changes in O/D ratios) as relevant mechanisms operating during the transient reduction in D-period, and are schematically 
shown at the intrafibrillar/molecular level in Fig. 5E. In contrast, analysis of the equatorial intermolecular spacing between tropocollagen molecules inside the fibrils shows no change during the D-period reduction (Supplementary Fig. S6A), indicating intrafibrillar loss of water to the extrafibrillar compartment is not a major factor. Further, analysis of the azimuthal distribution of SAXD intensity show that the fibril orientation distribution does not change during the transient reduction in D-period (Supplementary Fig. S6B). The data presented are thus supportive of mechanism (i) rather than mechanisms (ii)-(iii), where under compression, as a result of localised changes in swelling pressure, there is a transient reduction in fibrillar D-period accompanied by intrafibrillar disordering and changes in O/D ratio, followed by a reordering as the tissue equilibrates. A schematic of these mechanisms at the fibrillar level (one level higher than the intrafibrillar scale) is given in Fig. 6 .

The time delay (Figs. 2-4), from the start of relaxation to the time at which the transient changes in the D-period occurs, may be explained by the graded structure within cartilage. The depth-dependent poroelastic variation in extracellular matrix composition and organisation leads to a reduction in the compression induced deformation of the deeper zones compared to the superficial zone. ${ }^{41,42}$ Simulations to determine the localised mechanical environment within this inhomogeneous tissue have shown that drag forces between solid and fluid, fluid pressure and velocities, are zone-dependent, such that there are time-delayed peaks in the transitional and deep zone under timedependent loading. ${ }^{43,44}$ These increases in force and pressure will likely induce fluid 
flow out of the proteoglycan gel around the fibrils in a time-dependent manner such that there is a wave propagation of localised strain transfer. ${ }^{45}$

Our findings on the nanoscale mechanics of cartilage, including the reductions of fibrillar D-period due to removal of proteoglycan and associated water content, as well as the alterations in intrafibrillar ordering and molecular packing, can be linked to recent findings of hydration-induced alterations in fibrillar and intrafibrillar structure in tendon. ${ }^{3}$ Masic et al. found, in tendon collagen, that removal of water induced shifts in fibril Dperiod comparable to the pre-strains observed here ( $2.5 \%$ vs $1.5 \%$ in our case), and osmotic pressure changes had similar (but smaller) effects. ${ }^{3}$ Although the studies cannot be directly compared as Masic et al. ${ }^{3}$ studied complete dehydration of tendon collagen, while in our case we investigate partial loss of proteoglycans and associated water in cartilage collagen, the fibrillar-level structural changes in strain and molecular ordering may be related. Under a compositional change of the ECM (removal of $30-50 \%$ of proteoglycan ${ }^{47,48}$ ) that can be considered a disruptive change similar to but somewhat less severe than that induced by dehydration, ${ }^{3}$ stress relaxation in the fibrils is larger in articular cartilage than in tendon. This potentially reflects the greater volume fraction (and thus influence) of the non-fibrillar matrix in cartilage, as well as the difference in ECM architecture. Further, even during application of strains that are physiological in level (during stress-relaxation), the pre-strain reduction at the point of minimum Dperiod was $\sim 0.5 \%$, corresponding to a $2.5 \mathrm{MPa}$ stress off-loaded from the fibrils. Interestingly, the level of stress removed from the collagen fibrils in cartilage on enzymatic digestion ( 7.5 MPa) is considerably larger than the osmotic pressures of 
proteoglycans of $\sim 0.1-0.4 \mathrm{MPa}$ reported in Chahine et al. ${ }^{46}$ across solutions of varying ionic strength, with similar values reported by Maroudas and co-workers. ${ }^{35}$ Experiments where both PG content and ionic concentration are varied, together with the alteration of fibrillar pre-strain and structure, may in future shed light on these differences, as would selective removal of specific PG components like hyaluronan. We lastly note that these stress-values (such as the value of $7.5 \mathrm{MPa}$ reported earlier) are of the order of the maximum macroscopic compressive stresses in cartilage. For example, the maximum stress during stress relaxation in bovine cartilage, over similar percentage strains as in this study, can range from less than $1 \mathrm{MPa}$ (Supplementary Information, Fig. S2) to 6$8 \mathrm{MPa} .{ }^{15,49}$ Such large internal pre-stresses have been found in tendon collagen as well. $^{3}$ In this context, our results show clear evidence for the highly pre-stressed environment of the collagen fibrils in situ and in physiological conditions in cartilage, which may be related to recent suggestions concerning the active role of collagen fibrils in connective tissue. . $^{3,36}$

\section{Conclusion:}

To conclude, we have quantified the presence of pre-strain in cartilage collagen prior to compression. We also find that under physiological levels of compressive strain, the stress-relaxation dynamics of the collagen fibrils in articular cartilage shows an unusual behaviour which is not detected in the macroscopic mechanical response. This phenomenon appears as a delayed but very rapid reduction in collagen pre-strain followed by an equally rapid recovery. We suggest that the temporal changes in collagen strain and diffraction intensity are related to compression induced changes in 
water content in the extrafibrillar proteoglycan phase. These changes in the interfibrillar phase, which controls swelling pressure, lead to alterations in the intrafibrillar structure, specifically an increase in fibrillar disordering and a decrease in the overlap of adjacent axially staggered tropocollagen molecules. These mechanisms have implications for cartilage biomechanics and are likely to prove valuable for computational modelling, particularly in incorporating collagen fibrillar network mechanics (as per Fig. 6) in fibrilreinforced poroelastic models of cartilage, where up to now the mechanical behaviour of collagen has had to be assumed. ${ }^{29}$ Future studies may investigate the influence of different loading, disease and ageing conditions, which are all associated with changes in cartilage proteoglycan and water content. ${ }^{45,50}$ These factors are expected to regulate swelling and osmotic pressures as well as collagen D-period and microfibril packing, and significantly alter biomechanical performance, with biomedical and clinical implications in joint degeneration. While the details of the mechanism behind the delayed transient reduction in fibril strain are, as yet, not fully understood, we suggest that this interesting phenomenon may provide a step change in understanding collagen nano-mechanics, which is of fundamental importance in cartilage and other hydrated collagenous soft tissues.

\section{Materials and Methods:}

\section{Bovine explant preparation}

Bovine explants were extracted from the metacarpal-phalangeal joint of freshly slaughtered adult bovine steers (aged 18-24 months), which were obtained from a local abattoir. Full depth cartilage explants were isolated using $2 \mathrm{~mm}$ biopsy punches from the normal load bearing areas of the proximal surface of the joint. The explants were then 
cultured in Dulbecco's Modified Eagles Medium (DMEM) supplemented with 10\% fetal calf serum (FCS), 1.9mM L-glutamine, $96 \mathrm{U} / \mathrm{ml}$ penicillin, $96 \mathrm{mg} / \mathrm{ml}$ streptomycin (SigmaAldrich, Poole, UK). The explants were rested for 24 hours at $37^{\circ} \mathrm{C}, 5 \% \mathrm{CO}_{2}$, before being transferred into a 96-well plate. Explants were cultured for 24 hours either in normal media, Chondroitinase $A B C$ at $0.1 \mathrm{U} / \mathrm{ml}$ or Collagenase at $10 \mathrm{U} / \mathrm{ml}$ (SigmaAldrich, Poole, UK). Samples were snap frozen in liquid nitrogen followed by storage at $-20^{\circ} \mathrm{C}$ for subsequent mechanical testing and SAXD analysis.

\section{Human explant preparation}

Macroscopically normal human explants were isolated from the femoral condyle of a 44 Year old male cadaver supplied by Imperial College, London, UK. All procedures were conducted with full approval from the local ethics committee. As with the bovine explants, $2 \mathrm{~mm}$ explants were extracted using biopsy punches. Explants were maintained also in culture medium (DMEM $+10 \% \mathrm{FCS})$ for 24 hours, and then snap frozen in liquid nitrogen followed by storage at $-80^{\circ} \mathrm{C}$

\section{Mechanical Testing}

Mechanical tests were conducted on a subset of explants to determine efficacy of digestion as well as to pre-characterise the mechanics, prior to in situ SAXD measurements on the remaining samples. The same mechanical testing protocol was also used for testing during synchrotron SAXD measurements. The explant thickness was measured using calibrated Vernier callipers to within $10 \mu \mathrm{m}$. Explants were then compressed in unconfined conditions in a custom made micro-compression tester with 
a $22 \mathrm{~N}$ load cell (RDP Electronics, UK), and a LabVIEW control interface (National Instruments, UK). Explants were hydrated using phosphate buffered saline (PBS). A $0.1 \mathrm{~N}$ tare load was initially applied prior to uniaxial compression at a strain rate of $20 \% /$ min to a maximum strain of $20 \%$. After compression, the tissue was held at $20 \%$ strain for a stress relaxation period of 900 s. Tissue stiffness was quantified by the tangent modulus calculated from the linear portion of the stress-strain curve during the ramp-up compression phase of the test. A total of 9 samples were tested in each group.

\section{In situ small-angle X-ray diffraction (SAXD)}

SAXD measurements were carried out on the microfocus endstation at the 122 beamline at Diamond Light Source (DLS, Harwell, UK). The beam size was $15 \mu \mathrm{m}$ and the photon energy $14 \mathrm{keV}$. The micro-compression tester was mounted onto the microfocus platform to allow simultaneous compression of the tissue during X-ray measurements (Fig. 1a). SAXD patterns were recorded with a Pilatus P3-2M detector (Dectris, Villingen, $\mathrm{CH}$ ) with a pixel size of $172 \mu \mathrm{m}$ and a resolution of $1475 \times 1679$ pixels (horizontal $x$ vertical)) The sample to detector distance of $841.7 \pm 1.0 \mathrm{~mm}$ was calibrated using silver behenate (AgBe).

The transitional and deep zones for each sample were first identified using a 1D SAXD line-scan along the sample which revealed clear qualitative differences between the superficial, transitional and deep zones (Fig. 1c). SAXD measurements were carried out during relaxation of the tissue (either in the deep or transitional zone), in an automated manner using Python scripts integrated into GDA, the open source framework for data collection at DLS (http://www.opengda.org/). Specifically, SAXD 
measurements with short exposure times $(0.5 \mathrm{~s}$ per measurement point) were carried out during the stress relaxation. During the measurements, the micro-compression stage was shifted in the horizontal direction with respect to the beam, to expose different regions of the tissue within a $500 \mu \mathrm{m}$ window in order to minimise radiation damage to the tissue. Horizontal scanning was chosen so that the X-ray beam would remain in the zone of interest, as the cartilage tissue is approximately homogenous parallel to the tissue surface. As the majority of the relaxation in stress occurs over the first $\sim 150$ seconds, the time-interval between SAXD acquisitions was shorter (10 seconds) in this period and longer (60 seconds) afterward. The superficial zone was not measured when performing time-dependent tracking using the X-ray beam, due to the limited thickness of the superficial zone in cartilage as well as its large displacement on deformation.

To determine shifts in fibrillar D-period used to calculate fibrillar strain, the D-period for each SAXD frame was measured by azimuthally averaging the 2D SAXD intensity from $0^{\circ}$ to $360^{\circ}$ about the beam centre to obtain the integrated intensity profile I(q) (Fig. 1a,c). The integrated data I(q) was then fitted to a Gaussian with a linear background to obtain the peak position $q_{0}$, which is equal to $2 \pi / D$, where $D$ represents the fibrillar $D$-period. Custom Perl scripts integrated with the Gnuplot software (www.gnuplot.info) and Fit2D ${ }^{51}$ were used to carry out the integration and fitting for all the SAXD patterns in a batchmanner. When calculating fibrillar pre-strain (\% change in D-period) the reference Dperiod value was taken as the value in the uncompressed state. To calculate peak intensities of different meridional orders in the SAXD pattern (e.g. $I_{5}$ for the $5^{\text {th }}$ order 
peak), the peak area was estimated (up to a multiplicative constant $\sqrt{2 \pi}$ ) from the product of the fitted amplitude and width.

To calculate lateral tropocollagen molecule spacing $d_{\mathrm{m}}$ between the collagen fibrils, a cake-shaped sector from $q \sim 1.7 \mathrm{~nm}^{-1}$ to $\sim 7.2 \mathrm{~nm}^{-1}$ and oriented at $90^{\circ}$ to the (vertical) fibril direction was defined in Fit2D. After azimuthally averaging the intensity, and subtracting the diffuse SAXS background (Supplementary Fig. S5A), the equatorial SAXS peak was fitted to a Gaussian (Supplementary Fig. S5B) with peak position $q_{\mathrm{m}}$, and $d_{\mathrm{m}}$ was calculated from $d_{\mathrm{m}}=2 \pi / q_{\mathrm{m}}$.

To determine the ratio of the overlap zone $(\mathrm{O})$ in the fibril to the D-period (O/D here and elsewhere) from the SAXD pattern, in the presence of intrafibrillar disorder, an analysis approach combining elements of prior analysis ${ }^{36,37}$ is used. Specifically, in the absence of disorder, the ratio of the $m^{\text {th }}$ to $n^{\text {th }}$ order meridional Bragg peak intensities in the SAXD pattern is $\frac{I_{m}}{I_{n}}=\left(\frac{n}{m}\right)^{2}\left(\frac{\sin (m \pi(O / D))}{\sin (n \pi(O / D))}\right)^{2} \cdot 37,40$ If a degree of disordering of the sharp gap/overlap interface is present, the peak intensities are reduced by a DebyeWaller type factor $\exp \left(-\kappa q^{2}\right)$ where $\kappa$ is a term proportional to the disordering, and $q$ is the wavevector of the specific SAXD peak. ${ }^{36}$ Higher order peaks (with larger $q$ ) are thus reduced in intensity by a larger amount than lower order peaks. Combining these effects and using $q_{n}=\frac{2 \pi n}{D}$, we have Equations (1) and (2):

$\frac{I_{7}}{I_{5}}=\left(\frac{5}{7}\right)^{2}\left(\frac{\sin (7 \pi(O / D))}{\sin (5 \pi(O / D))}\right)^{2} \exp \left(-24 \kappa\left(\frac{2 \pi}{D}\right)^{2}\right)$ Equation (1)

and 


$$
\frac{I_{8}}{I_{5}}=\left(\frac{5}{8}\right)^{2}\left(\frac{\sin (8 \pi(O / D))}{\sin (5 \pi(O / D))}\right)^{2} \exp \left(-39 \kappa\left(\frac{2 \pi}{D}\right)^{2}\right) \quad \text { Equation (2) }
$$

In the Supplementary Information, the variation of $\frac{I_{7}}{I_{5}}$ and $\frac{I_{8}}{I_{5}}$ is plotted for different levels of disorder (Fig. S4), and the calculation of O/D and $\kappa$ from known values of $\frac{I_{7}}{I_{5}}$ and $\frac{I_{8}}{I_{5}}$ is described in Supplementary Information Section S2.

\section{Statistical Analysis}

The representative trace refers to a single sample whereas the grouped data are mean values with standard error of mean (SEM) (where $n=5-9)$. Statistical analyses were performed using Microsoft Excel. Data was analysed and significance measured using a either paired (within-group) or un-paired (between-groups) t-test. Within-group tests refer to statistical significance relative to the corresponding uncompressed fibrillar state (prior to mechanical loading) within a sample group and are indicated at $p<0.05$ $(\#), \quad p<0.01$ (\#\#) and $p<0.001(\# \#)$. Between-group tests refer to the statistical significance of the enzymatic group relative to the control groups within each fibrillar state and are indicated at $p<0.05\left(^{*}\right), p<0.01\left(^{* *}\right)$ and $p<0.001\left(^{* * *}\right)$.

\section{Acknowledgements}

Sheetal R. Inamdar is supported by an Institute of Bioengineering (QMUL) PhD studentship (funded through the EPSRC). We thank Diamond Light Source (Harwell, UK) for the generous award of synchrotron beamtime (SM10311).The studies were partly supported by an MRC project grant (MR/L002876/1: PI Knight, 'Osteoarthritis may 
be treated as an environmental ciliopathy').We are grateful to Humphrey's \& Sons the local abattoir for supply of bovine metacarpal phalangeal joints. A. Karunaratne thanks Anthony Bull (Department of Bioengineering, Imperial College, London) for the supply of human tissue samples. Tissue samples were provided by the Imperial College Healthcare NHS Trust Tissue Bank (ICHTB). Other investigators may have received samples from these same tissues. The research was supported by the National Institute for Health Research (NIHR) Biomedical Research Centre based at Imperial College Healthcare NHS Trust and Imperial College London (Project R15046). The views expressed are those of the author(s) and not necessarily those of the NHS, the NIHR or the Department of Health. We would like to thank the anonymous reviewers for the suggestion to analyse intensity ratio and equatorial SAXS peak changes for insight into the mechanisms.

\section{AUTHOR INFORMATION}

\section{Corresponding Author}

${ }^{*}$ Dr. Himadri S. Gupta, School of Engineering and Materials Science and Institute of Bioengineering, Queen Mary University of London, Mile End Road, London E1 4NS. Email: h.gupta@qmul.ac.uk.

\section{Present Addresses}

†Current address: Department of Mechanical Engineering, University of Moratuwa, Sri Lanka.

\section{Author Contributions}


The manuscript was written through contributions of all authors. All authors have given approval to the final version of the manuscript.

\author{
Funding Sources \\ MRC project grant (MR/L002876/1); National Institute for Health Research (NIHR \\ $\mathrm{R} 15046)$.
}

\title{
Description of Supporting Information Available Online
}

Supporting Information is available online, consisting of three subsections. S.1 shows data on SGAG release and mechanical alterations with chondroitinase treatment. S.2 provides details on modelling of SAXD intensity changes during stress relaxation in terms of fibrillar structural changes. S.3 contains data on intrafibrillar molecular packing and fibril orientation during stress relaxation. 


\section{Figure Captions:}

Figure 1. Experimental setup for in situ structural analysis of collagen fibrils in cartilage. (A) A micro-compression tester was used in line with the x-ray beam to simultaneously measure changes in fibril strain whilst performing stress relaxation tests. (B) Representative SAXD pattern from bovine articular cartilage. A combination of fibrils at different predominant angles contributes to the diffraction peaks. Due to the periodic electron density along the collagen fibril axis (with a period D), a set of Bragg peaks at multiples of $2 \pi / D$ could be observed within the X-ray scattering patterns. (C) The depthdependent collagen architecture in articular cartilage can be observed in the associated diffraction patterns, with fibrillar D-period and orientation determined from the peak positions in the azimuthally and radially integrated intensity profiles, respectively.

Figure 2. Collagen fibrils experience a delayed reduction and recovery in fibrillar pre-strain in response to stress-relaxation. (a) Representative, macro-scale stress response in compressed bovine cartilage during relaxation (20\% strain level loaded at a rate of $20 \% / \mathrm{min}$ ). (b) Corresponding absolute and percentage change in D-period, relative to the unloaded local D-period. Red arrow highlights the time at minimum Dperiod. The onset of the event starts at $\sim 50$ seconds after peak load, and the subsequent D-period recovery is complete by $\sim 150$ seconds (Fig. 2b), during which period no visible changes in tissue stress is visible (Fig. 2a, inset).

Figure 3. Delayed fibrillar response to loading observed in human femoral cartilage. Percentage change in fibrillar D-period during macroscale tissue relaxation found in both the transitional (a) and deep (b) zones, averaged over multiple samples (Transitional $n=5$, Deep $n=4$ ). A delayed rapid reduction to a minimum D-period (red 
arrow), followed by a recovery is observed $\sim 50-100$ seconds after peak load in both cases. (c) The time from the start of tissue-level relaxation until the minimum D-period value; no significant differences were observed between the two zones $(p>0.05)$. (d) Variation in absolute D-period at different time points (uncompressed, at peak load, at the point of minimum $D$ and relaxed) for both transitional and deep zones. Error bars represent standard error of mean throughout, * indicate significance between groups, \# indicate significance within groups.

Figure 4. Enzymatic degradation leads to an altered fibrillar pre-strain alongside changes to fibrillar response directly after loading in bovine cartilage. Data shown separately for transitional $(a, c, e)$ and deep $(b, d, f)$ zones. $(a, b)$ Time-dependent variation in percentage change of D-period, showing delayed fibrillar response within both the transitional and deep zones in the control group and a loss of response in the transitional zone of the enzymatic group (a, lower plot). $(c, d)$ Time from the start of macro-scale relaxation to minimum D-period. $(e, f)$ Variations in absolute values of Dperiod (at the different stages of stress relaxation) in both the transitional and deep zones. These values show reduced fibrillar pre-strain as a result of enzymatic digestion. Error bars represent standard error of mean $(n=5),{ }^{*}$ indicate significance between groups, \# indicate significance within groups.

Figure 5. Intra- and interfibrillar structural alterations during transient reduction of pre-strain: A) The time-variation of the $7^{\text {th }}$ to the $5^{\text {th }}$ order peak intensity ratio $17 / I_{5}$ (solid black line) shows a characteristic dip (highlighted in dashed box) near the minimum in D (grey line); data from the sample shown in Fig. 2. B) Similar to A), but for the $8^{\text {th }}$ to the $5^{\text {th }}$ order peak intensity ratio $I_{8} / I_{5}$ and showing a similar dip near the 
minimum in D. C) Left: A temporally magnified overlay of the D-period variation (gray line), $I_{7} / I_{5}$ (open symbols) and $I_{8} / I_{5}$ (filled symbols) near the minimum in $D$, showing approximately three distinct regions: (1) both $I_{7} / I_{5}$ and $I_{8} / I_{5}$ reduce from their initial values of $\sim 0.25$ and $\sim 0.15$, in parallel with the reduction in $D$. (2) $D$ continues to reduce, reaches the minimum and increases, but $I_{7} / I_{5}$ and $I_{8} / I_{5}$ vary in opposite directions: $I_{8} / I_{5}$ increases followed by a levelling off, whilst $I_{7} / I_{5}$ decreases followed by a later increase. The symbols $(a, b, c, d)$ denote specific time-demarcation points, and are explained in part D). Right: Schematic of the fibrillar level changes which can be inferred from the changes in the left panel, specifically a combination of intrafibrillar disordering and change in overlap/D ratio, as will be made quantitative in D). D) Modelling of the observed behavior in $\mathrm{C}$ ), in terms of changes in overlap/D ratio and changes in the intrafibrillar disorder parameter $\kappa$. Lines indicate plots of predicted $I_{7} / I_{5}$ (blue) and $I_{8} / I_{5}$ (red) for varying overlap/D ratio (abscissa), using a step-function model (with intrafibrillar disorder) for SAXD intensity. Different line styles indicate varying levels of intrafibrillar disorder; dashed: $\kappa=0.00 \mathrm{~nm}^{2}$ (no disorder), solid: $\kappa=\kappa_{0}=1.75 \mathrm{~nm}^{2}$, dashdot: $\kappa=\kappa_{1}=4.0 \mathrm{~nm}^{2}$. Circles indicate different stages $(a \rightarrow b \rightarrow c \rightarrow d)$ in the process, which correspond to the times in $C)$, where the labels $O(a-d)$ and $E(a-d)$ refer to the odd $\left(I_{7} / I_{5}\right)$ and even $\left(I_{8} / I_{5}\right)$ order ratio values at each stage respectively. An increase in disorder (vertical down arrow; $a \rightarrow b$ ) is followed by a change in O/D ratio (left-inclined arrow; $b \rightarrow c)$, concluded by a return to near-initial values $(c \rightarrow d)$. Arrows are displaced laterally in the $(a \rightarrow b)$ stage to avoid overlap. E) Schematics of the intrafibrillar-level mechanisms corresponding to the transient changes in D. Each vertical dark blue rod corresponds to a single tropocollagen molecule, which are aggregated to form a fibril. 
D-period, overlap (O) and gap $(\mathrm{G})$ regions indicated on the leftmost schematic. As above, $a, b, c$ and $d$ correspond to points in Fig. 5D. Left to right: ordered arrangement of tropocollagen molecules with well-defined gap/overlap interface, followed by an increase in intrafibrillar disorder leading to a blurred interface, then a reduction in overlap zone and finally ordered intrafibrillar arrangement after recovery of pre-strain for schematic clarity, the residual disorder at the last stage is not depicted.

Figure 6. Fibrillar level mechanisms underlying transient change in pre-strain in cartilage: (A) Schematic of the cartilage ECM nanostructure at the fibrillar $(\sim 10-100 \mathrm{~nm})$ scale, with ordered Type II collagen fibrils (banded rods) surrounded by swollen, amorphous aggregates of negatively charged proteoglycans (orange circles) with a large number of loosely bound water molecules (blue circles)). Orange arrows indicated direction and relative magnitude of tensile pre-strain exerted by the proteoglycan aggregates on the collagen fibril. Left: Unloaded cartilage. Middle: Static compression of tissue is followed by a transient reduction of pre-strain in the collagen fibrils, possibly due to loss of water molecules and structural collapse in the proteoglycan network (blue arrows indicate direction of fluid flow). The reduction in pre-strain is shown by a decrease in D-period. In addition the gap/overlap interface in the collagen D-period banding pattern becoming increasingly blurred, suggesting an associated dis-ordering and loss of crystallinity in the collagen molecules. Right: As water molecules return to the proteoglycan aggregates there is a restoration of collagen fibril pre-strain and ordering/crystallinity. 


\section{References:}

(1) Bakarich, S. E.; Gorkin, R.; in het Panhuis, M.; Spinks, G. M. Three-Dimensional Printing Fiber Reinforced Hydrogel Composites. ACS Appl. Mater. Interfaces 2014, 6, 15998-16006.

(2) Wu, Z. L.; Moshe, M.; Greener, J.; Therien-Aubin, H.; Nie, Z.; Sharon, E.; Kumacheva, E. Three-Dimensional Shape Transformations of Hydrogel Sheets Induced by Small-Scale Modulation of Internal Stresses. Nat. Commun. 2013, 4, 1586.

(3) Masic, A.; Bertinetti, L.; Schuetz, R.; Chang, S.-W.; Metzger, T. H.; Buehler, M. J.; Fratzl, P. Osmotic Pressure Induced Tensile Forces in Tendon Collagen. Nat. Commun. 2015, 6, 5942.

(4) Stamenovic, D.; Ingber, D. E. Tensegrity-Guided Self Assembly: From Molecules to Living Cells. Soft Matter 2009, 5, 1137-1145.

(5) Gardel, M. L.; Shin, J. H.; MacKintosh, F. C.; Mahadevan, L.; Matsudaira, P. A.; Weitz, D. A. Scaling of F-Actin Network Rheology to Probe Single Filament Elasticity and Dynamics. Phys. Rev. Lett. 2004, 93.

(6) Guilak, F. Biomechanical Factors in Osteoarthritis. Best Pract. Res., Clin. Rheumatol. 2011, 25, 815-823.

(7) Broom, N. D.; Silyn-Roberts, H. Collagen-Collagen versus Collagen-Proteoglycan Interactions in the Determination of Cartilage Strength. Arthritis Rheum. 1990, 33, $1512-1517$. 
(8) Setton, L. A.; Elliott, D. M.; Mow, V. C. Altered Mechanics of Cartilage with Osteoarthritis: Human Osteoarthritis and an Experimental Model of Joint Degeneration. Osteoarthr. Cartil. 1999, 7, 2-14.

(9) Mansour, J. M.; Ph, D. Biomechanics of Cartilage. Kinesiol. Mech. Pathomechanics Hum. Mov. 2003, 66-79.

(10) Korhonen, R. K.; Laasanen, M. S.; Töyräs, J.; Rieppo, J.; Hirvonen, J.; Helminen, H. J.; Jurvelin, J. S. Comparison of the Equilibrium Response of Articular Cartilage in Unconfined Compression, Confined Compression and Indentation. J. Biomech. 2002, 35, 903-909.

(11) Responte, D. J.; Natoli, R. M.; Athanasiou, K. A. Colllagens of Articular Cartilage.pdf. Crit. Rev. Biomed. Eng. 2007, 35, 363-411.

(12) Kleeman, R. U.; Krocker, D.; Cedrano, A.; Tuischer, J.; Duda, G. N. Altered Cartilage Mechanics and Histology in Knee Osteoarthritis: Relation to Clinical Assessment (ICRS Grade). Osteoarthr. Cartil. 2005, 13, 958-963.

(13) Han, L.; Grodzinsky, A. J.; Ortiz, C. Nanomechanics of the Cartilage Extracellular Matrix. Annu. Rev. Mater. Res. 2011, 41, 133-168.

(14) Han, E.; Chen, S. S.; Klisch, S. M.; Sah, R. L. Contribution of Proteoglycan Osmotic Swelling Pressure to the Compressive Properties of Articular Cartilage. Biophys. J. 2011, 101, 916-924.

(15) Korhonen, R. K.; Jurvelin, J. S. Compressive and Tensile Properties of Articular Cartilage in Axial Loading Are Modulated Differently by Osmotic Environment. 
Med. Eng. Phys. 2010, 32, 155-160.

(16) Potter, K.; Kidder, L. H.; Levin, I. W.; Lewis, E. N.; Spencer, R. G. S. Imaging of Collagen and Proteoglycan in Cartilage Sections Using Fourier Transform Infrared Spectral Imaging. Arthritis. Rheum. 2001, 44, 846-855.

(17) Bi, X.; Li, G.; Doty, S. B.; Camacho, N. P. A Novel Method for Determination of Collagen Orientation in Cartilage by Fourier Transform Infrared Imaging Spectroscopy (FT-IRIS). Osteoarthr. Cartil. 2005, 13, 1050-1058.

(18) Changoor, A.; Nelea, M.; Méthot, S.; Tran-Khanh, N.; Chevrier, A.; Restrepo, A.; Shive, M. S.; Hoemann, C. D.; Buschmann, M. D. Structural Characteristics of the Collagen Network in Human Normal, Degraded and Repair Articular Cartilages Observed in Polarized Light and Scanning Electron Microscopies. Osteoarthr. Cartil. 2011, 19, 1458-1468.

(19) Gupta, H. S.; Seto, J.; Krauss, S.; Boesecke, P.; Screen, H. R. C. In Situ MultiLevel Analysis of Viscoelastic Deformation Mechanisms in Tendon Collagen. J. Struct. Biol. 2010, 169, 183-191.

(20) Puxkandl, R.; Zizak, I.; Paris, O.; Keckes, J.; Tesch, W.; Bernstorff, S.; Purslow, P.; Fratzl, P. Viscoelastic Properties of Collagen: Synchrotron Radiation Investigations and Structural Model. Philos. Trans. R. Soc., B 2002, 357, 191197.

(21) Karunaratne, A.; Esapa, C. R.; Hiller, J.; Boyde, A.; Head, R.; Bassett, J. H. D.; Terrill, N. J.; Williams, G. R.; Brown, M. A; Croucher, P. I.; Brown, S. D. M.; Cox, 
R. D.; Barber, A. H.; Thakker, R. V.; Gupta, H. S. Significant Deterioration in Nanomechanical Quality Occurs through Incomplete Extrafibrillar Mineralization in Rachitic Bone: Evidence from In-Situ Synchrotron X-Ray Scattering and Backscattered Electron Imaging. J. Bone Miner. Res. 2012, 27, 876-890.

(22) Zimmermann, E. A.; Schaible, E.; Bale, H.; Barth, H. D.; Tang, S. Y.; Reichert, P.; Busse, B.; Alliston, T.; Ager 3rd, J. W.; Ritchie, R. O. Age-Related Changes in the Plasticity and Toughness of Human Cortical Bone at Multiple Length Scales. Proc. Natl. Acad. Sci. U. S. A. 2011, 108, 14416-14421.

(23) Almer, J. D.; Stock, S. R. Internal Strains and Stresses Measured in Cortical Bone via High-Energy X-Ray Diffraction. J. Struct. Biol. 2005, 152, $14-27$.

(24) Yang, W.; Sherman, V. R.; Gludovatz, B.; Schaible, E.; Stewart, P.; Ritchie, R. O.; Meyers, M. A. On the Tear Resistance of Skin. Nat. Commun. 2015, 6, 6649.

(25) Aspden, R. ; Hukins, D. W. Collagen Organization in Articular Cartilage, Determined by X-Ray Diffraction, and Its Relationship to Tissue Function. Proc. $R$. Soc. Lond., Ser. B 1981, 212, 299-304.

(26) Moger, C. J.; Arkill, K. P.; Barrett, R.; Bleuet, P.; Ellis, R. E.; Green, E. M.; Winlove, C. P. Cartilage Collagen Matrix Reorientation and Displacement in Response to Surface Loading. J. Biomech. Eng. 2009, 131, 31008.

(27) Moger, C. J.; Barrett, R.; Bleuet, P.; Bradley, D. a; Ellis, R. E.; Green, E. M.; Knapp, K. M.; Muthuvelu, P.; Winlove, C. P. Regional Variations of Collagen Orientation in Normal and Diseased Articular Cartilage and Subchondral Bone 
Determined Using Small Angle X-Ray Scattering (SAXS). Osteoarthr. Cartil. 2007, $15,682-687$.

(28) Mollenhauer, J.; Aurich, M.; Muehleman, C.; Khelashvilli, G.; Irving, T. C. X-Ray Diffraction of the Molecular Substructure of Human Articular Cartilage. Connect. Tissue Res. 2003, 44, 201-207.

(29) Julkunen, P.; Wilson, W.; Isaksson, H.; Jurvelin, J. S.; Herzog, W.; Korhonen, R. K. A Review of the Combination of Experimental Measurements and FibrilReinforced Modeling for Investigation of Articular Cartilage and Chondrocyte Response to Loading. Comput. Math. Methods Med. 2013, 2013, 326150.

(30) Shirazi, R.; Shirazi-Adl, a; Hurtig, M. Role of Cartilage Collagen Fibrils Networks in Knee Joint Biomechanics under Compression. J. Biomech. 2008, 41, 33403348.

(31) Ateshian, G. A.; Rajan, V.; Chahine, N. O.; Canal, C. E.; Hung, C. T. Modeling the Matrix of Articular Cartilage Using a Continuous Fiber Angular Distribution Predicts Many Observed Phenomena. J. Biomech. Eng. 2009, 131, 61003.

(32) Eppell, S. J.; Smith, B. N.; Kahn, H.; Ballarini, R. Nano Measurements with MicroDevices: Mechanical Properties of Hydrated Collagen Fibrils. J. R. Soc., Interface 2006, 3, 117-121.

(33) Xia, Y.; Momot, K.; Chen, Z.; Chen, C.; Kahn, D.; Badar, F. Introduction to Cartilage. In Biophysics and Biochemistry of Cartilage by NMR and MRI; Xia, Y.; Momot, K., Eds.; The Royal Society of Chemistry: Cambridge, 2017; pp. 3-35. 
(34) Nordin, M.; Frankel, V. Basic Biomechanics of the Musculoskeletal System; 3rd ed.; Lippincott Williams \& Wilkins, 2001.

(35) Wachtel, E.; Maroudas, A. The Effects of $\mathrm{pH}$ and Ionic Strength on Intrafibrillar Hydration in Articular Cartilage. Biochim. Biophys. Acta, Gen. Subj. 1998, 1381, $37-48$.

(36) Fratzl, P.; Misof, K.; Zizak, I.; Rapp, G.; Amenitsch, H.; Bernstorff, S. Fibrillar Structure and Mechanical Properties of Collagen. J. Struct. Biol. 1997, 122, 119 122.

(37) Mosler, E.; Folkhard, W.; Knörzer, E.; Nemetschek-Gansler, H.; Nemetschek, T.; Koch, M. H. Stress-Induced Molecular Rearrangement in Tendon Collagen. J. Mol. Biol. 1985, 182, 589-596.

(38) Fratzl, P. Collagen Packing and Mineralization: An X-Ray Scattering Investigation of Turkey Leg Tendon. Biophys. J. 1993, 64, 260-266.

(39) Lees, S.; Bonar, L. C.; Mook, H. A. A Study of Dense Mineralized Tissue by Neutron Diffraction. Int. J. Biol. Macromol. 1984, 6, 321-326.

(40) Sasaki, N.; Odajima, S. Elongation Mechanisms of Collagen Fibrils and ForceStrain Relations of Tendons at Each Level of Structural Hierarchy. J. Biomech. 1996, 29, 1131-1136.

(41) Erne, O. K.; Reid, J. B.; Ehmke, L. W.; Sommers, M. B.; Madey, S. M.; Bottlang, M. Depth-Dependent Strain of Patellofemoral Articular Cartilage in Unconfined Compression. J. Biomech. 2005, 38, 667-672. 
(42) Halonen, K. S.; Mononen, M. E.; Jurvelin, J. S.; Töyräs, J.; Korhonen, R. K. Importance of Depth-Wise Distribution of Collagen and Proteoglycans in Articular Cartilage-A 3D Finite Element Study of Stresses and Strains in Human Knee Joint. J. Biomech. 2013, 46, 1184-1192.

(43) Wang, C. C. B.; Hung, C. T.; Mow, V. C. An Analysis of the Effects of DepthDependent Aggregate Modulus on Articular Cartilage Stress-Relaxation Behavior in Compression. J. Biomech. 2001, 34, 75-84.

(44) Setton, L. A.; Zhu, W.; Mow, V. C. The Biphasic Poroviscoelastic Behavior of Articular Cartilage: Role of the Surface Zone in Governing the Compressive Behavior. J. Biomech. 1993, 26, 581-592.

(45) Tavakoli Nia, H.; Han, L.; Soltani Bozchalooi, I.; Roughley, P.; Youcef-Toumi, K.; Grodzinsky, A. J.; Ortiz, C. Aggrecan Nanoscale Solid-Fluid Interactions Are a Primary Determinant of Cartilage Dynamic Mechanical Properties. ACS Nano 2015, 9, 2614-2625.

(46) Chahine, N. O.; Chen, F. H.; Hung, C. T.; Ateshian, G. A. Direct Measurement of Osmotic Pressure of Glycosaminoglycan Solutions by Membrane Osmometry at Room Temperature. Biophys. J. 2005, 89, 1543-1550.

(47) Roman, N. M.; Christopher, R. M.; Athanasiou, K. A. Chondroitinase ABC Treatment Results in Greater Tensile Properties of Self-Assembled TissueEngineered Articular Cartilage. Tissue Eng. 2009, 15.

(48) Korhonen, R. K.; Laasanen, M. S.; Töyräs, J.; Lappalainen, R.; Helminen, H. J.; 
Jurvelin, J. S. Fibril Reinforced Poroelastic Model Predicts Specifically Mechanical Behavior of Normal, Proteoglycan Depleted and Collagen Degraded Articular Cartilage. J. Biomech. 2003, 36, 1373-1379.

(49) Mow, V. C.; Guo, X. E. Mechano-Electrochemical Properties Of Articular Cartilage: Their Inhomogeneities and Anisotropies. Annu. Rev. Biomed. Eng. 2002, 4, 175-209.

(50) Pearle, A. D.; Warren, R. F.; Rodeo, S. A. Basic Science of Articular Cartilage and Osteoarthritis. Clin. Sports Med. 2005, 24, 1-12.

(51) Hammersley, A. P. FIT2D: A Multi-Purpose Data Reduction, Analysis and Visualization Program. J. Appl. Crystallogr. 2016, 49, 646-652. 

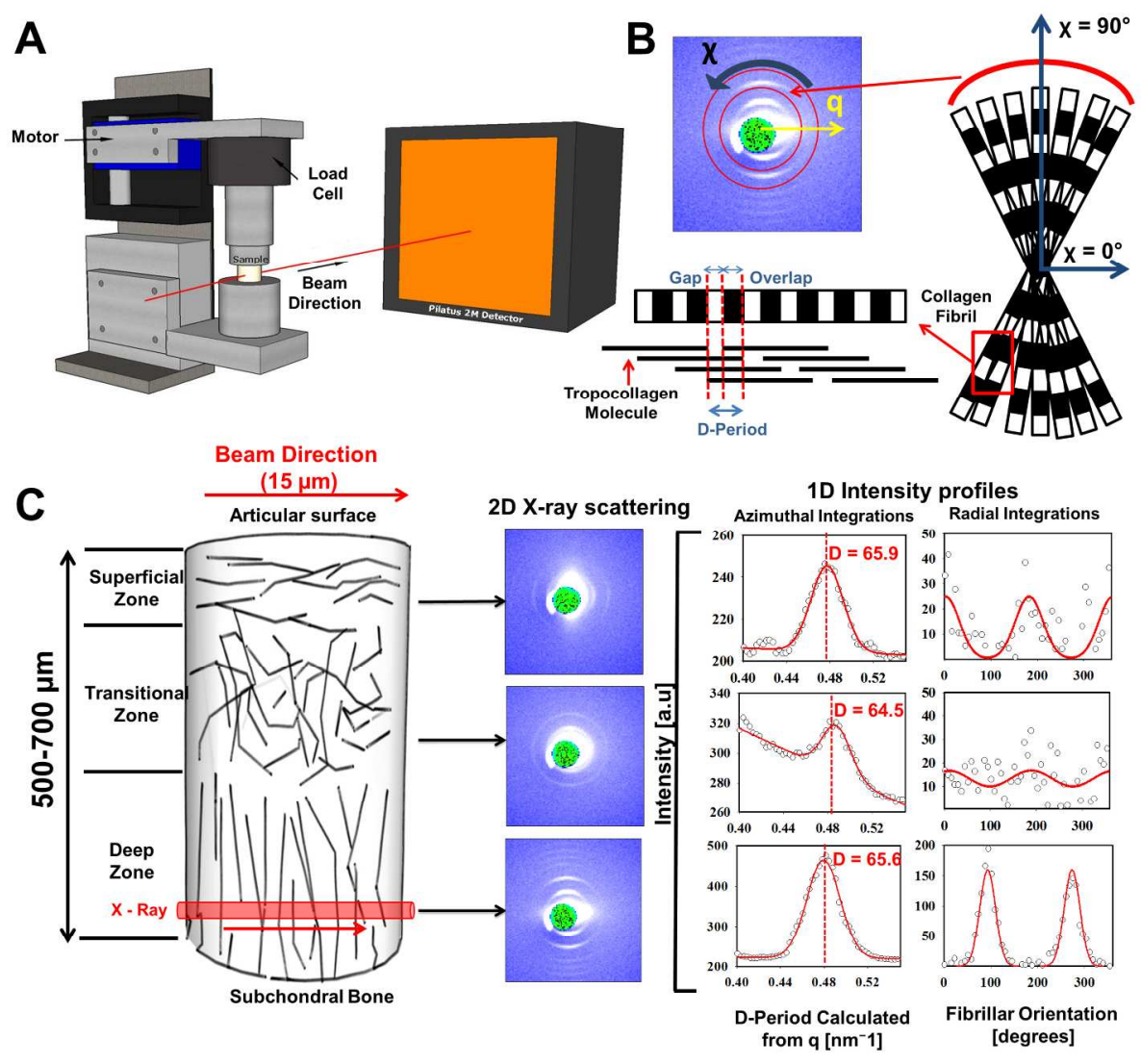

Figure 1. Experimental setup for in situ structural analysis of collagen fibrils in cartilage. (A) A micro-compression tester was used in line with the $x$-ray beam to simultaneously measure changes in fibril strain whilst performing stress relaxation tests. (B) Representative SAXD pattern from bovine articular cartilage. A combination of fibrils at different predominant angles contributes to the diffraction peaks. Due to the periodic electron density along the collagen fibril axis (with a period D), a set of Bragg peaks at multiples of $2 \pi / D$ could be observed within the X-ray scattering patterns. (C) The depth-dependent collagen architecture in articular cartilage can be observed in the associated diffraction patterns, with fibrillar Dperiod and orientation determined from the peak positions in the azimuthally and radially integrated intensity profiles, respectively.

$478 \times 411 \mathrm{~mm}(300 \times 300$ DPI) 

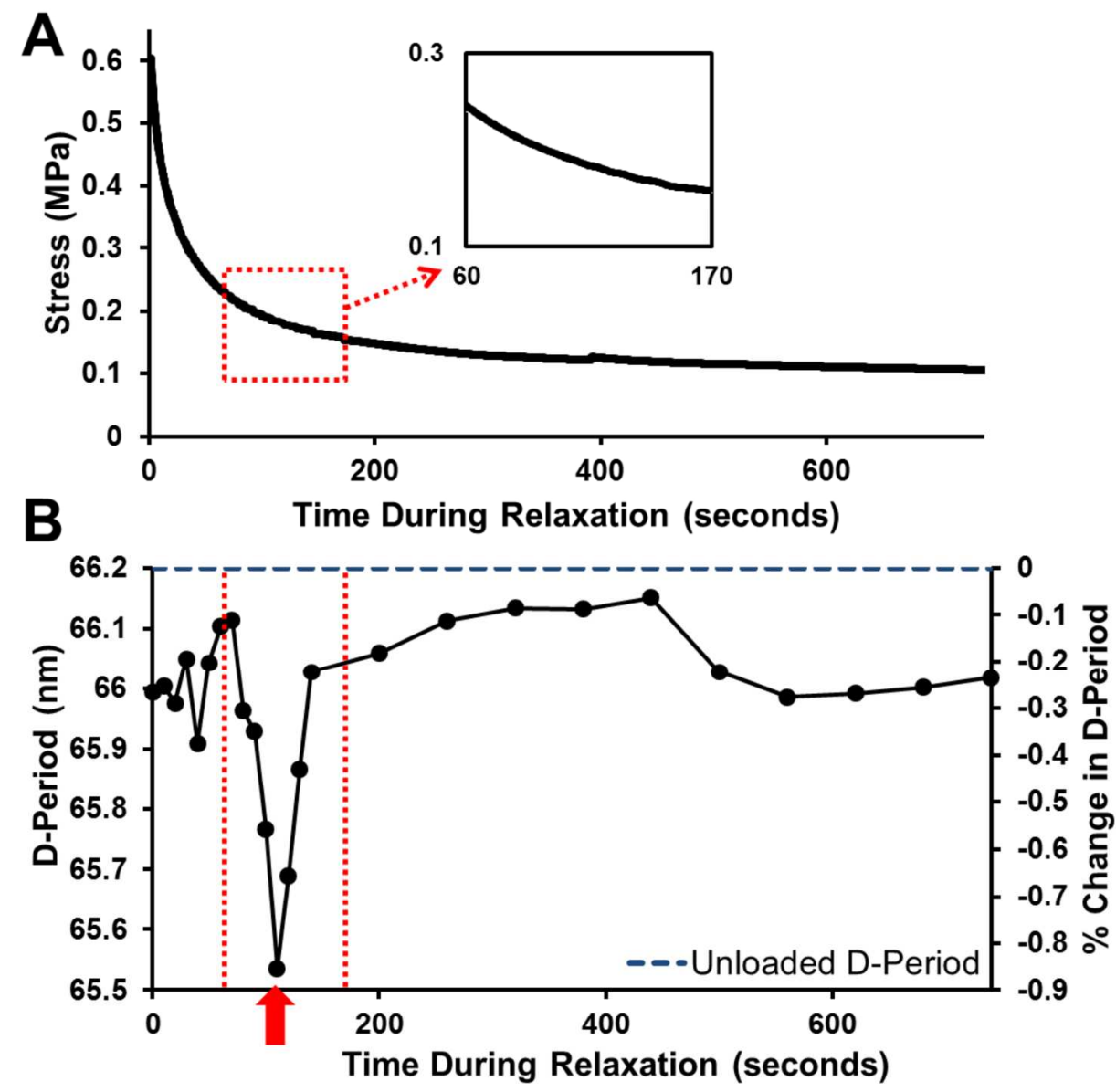

Figure 2. Collagen fibrils experience a delayed reduction and recovery in fibrillar pre-strain in response to stress-relaxation. (a) Representative, macro-scale stress response in compressed bovine cartilage during relaxation ( $20 \%$ strain level loaded at a rate of $20 \% / \mathrm{min}$ ). (b) Corresponding absolute and percentage change in D-period, relative to the unloaded local D-period. Red arrow highlights the time at minimum D-period. The onset of the event starts at $\sim 50$ seconds after peak load, and the subsequent $D$ period recovery is complete by $\sim 150$ seconds (Fig. 2b), during which period no visible changes in tissue stress is visible (Fig. 2a, inset).

$196 \times 190 \mathrm{~mm}(300 \times 300 \mathrm{DPI})$ 
Figure 3. Delayed fibrillar response to loading observed in human femoral cartilage. Percentage change in fibrillar D-period during macroscale tissue relaxation found in both the transitional (a) and deep (b) zones, averaged over multiple samples (Transitional $n=5$, Deep $n=4$ ). A delayed rapid reduction to a minimum D-period (red arrow), followed by a recovery is observed 50-100 seconds after peak load in both cases. (c) The time from the start of tissue-level relaxation until the minimum D-period value; no significant differences were observed between the two zones $(p>0.05)$. (d) Variation in absolute D-period at different time points (uncompressed, at peak load, at the point of minimum $D$ and relaxed) for both transitional and deep zones. Error bars represent standard error of mean throughout, * indicate significance between groups, \# indicate significance within groups.

\section{$292 \times 292 \mathrm{~mm}(300 \times 300$ DPI $)$}




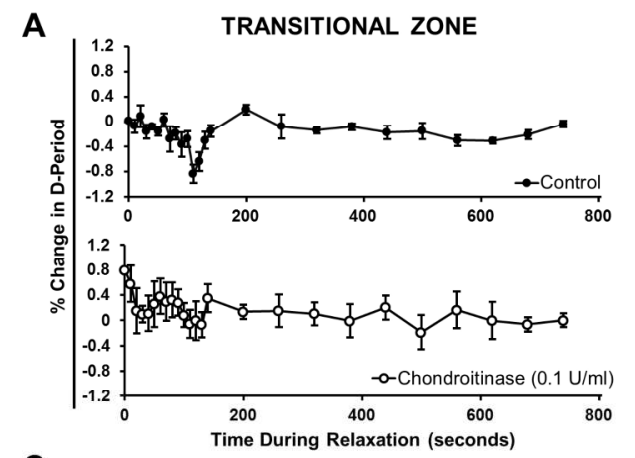

C

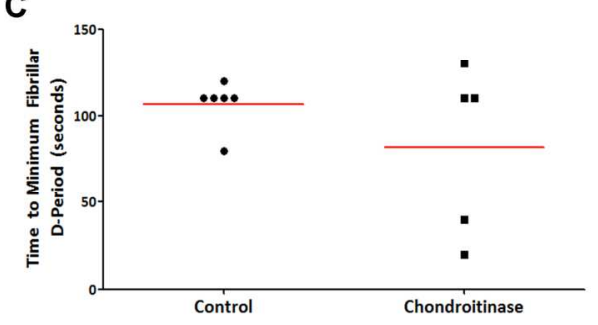

E

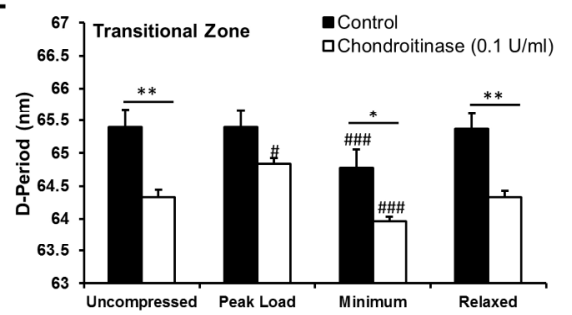

B
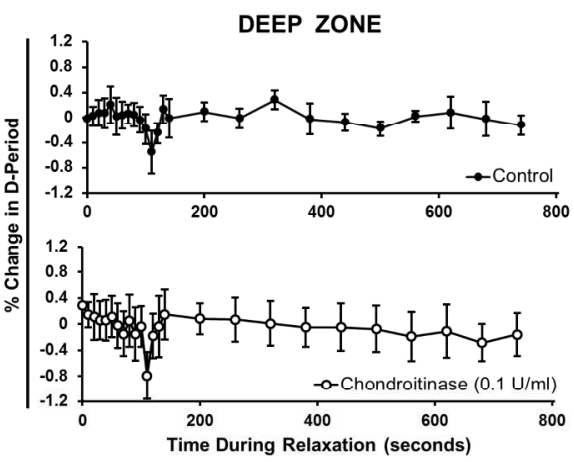

D

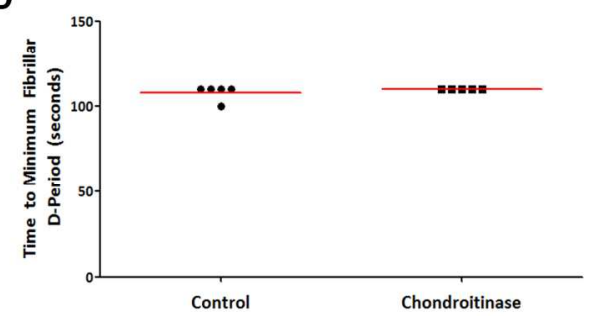

$\mathbf{F}$

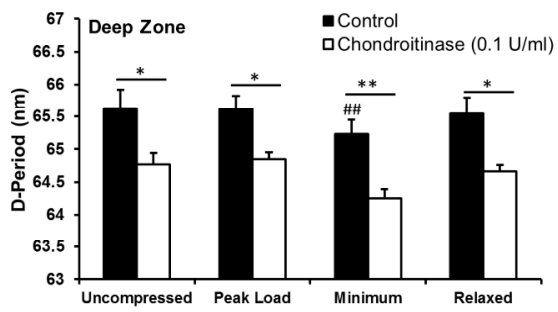

Figure 4. Enzymatic degradation leads to an altered fibrillar pre-strain alongside changes to fibrillar response directly after loading in bovine cartilage. Data shown separately for transitional $(a, c, e)$ and deep $(b, d, f)$ zones. $(a, b)$ Time-dependent variation in percentage change of $D$-period, showing delayed fibrillar response within both the transitional and deep zones in the control group and a loss of response in the transitional zone of the enzymatic group (a, lower plot). (c,d) Time from the start of macroscale relaxation to minimum $D$-period. $(e, f)$ Variations in absolute values of $D$-period (at the different stages of stress relaxation) in both the transitional and deep zones. These values show reduced fibrillar pre-strain as a result of enzymatic digestion. Error bars represent standard error of mean $(n=5)$, * indicate significance between groups, \# indicate significance within groups.

$$
387 \times 353 \mathrm{~mm} \text { ( } 300 \times 300 \text { DPI) }
$$


A
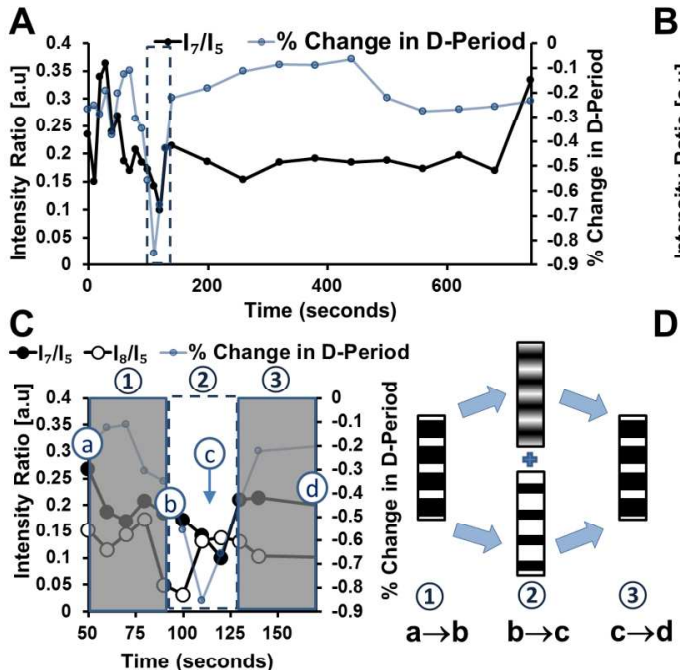

B

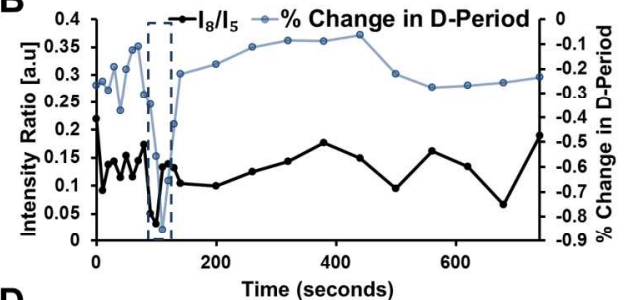

D

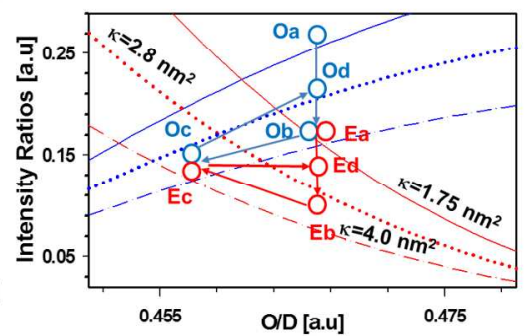

E
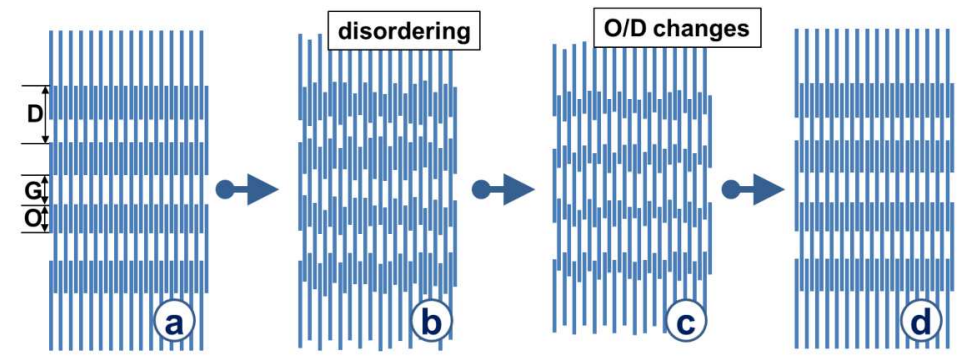

Figure 5. Intra- and interfibrillar structural alterations during transient reduction of pre-strain: $\mathrm{A}$ )

The time-variation of the 7th to the 5th order peak intensity ratio I7/I5 (solid black line) shows a characteristic dip (highlighted in dashed box) near the minimum in D (grey line); data from the sample shown in Fig. 2. B) Similar to A), but for the 8th to the 5th order peak intensity ratio I8/I5 and showing a similar dip near the minimum in D. C) Left: A temporally magnified overlay of the D-period variation (gray line), I7/I5 (open symbols) and I8/I5 (filled symbols) near the minimum in D, showing approximately three distinct regions: (1) both I7/I5 and I8/I5 reduce from their initial values of $\sim 0.25$ and $\sim 0.15$, in parallel with the reduction in D. (2) D continues to reduce, reaches the minimum and increases, but I7/I5 and I8/I5 vary in opposite directions: I8/I5 increases followed by a levelling off, whilst I7/I5 decreases followed by a later increase. The symbols ( $a, b, c, d)$ denote specific time-demarcation points, and are explained in part D). Right: Schematic of the fibrillar level changes which can be inferred from the changes in the left panel, specifically a combination of intrafibrillar disordering and change in overlap/D ratio, as will be made quantitative in D). D) Modelling of the observed behavior in C), in terms of changes in overlap/D ratio and changes in the intrafibrillar disorder parameter $\mathrm{K}$. Lines indicate plots of predicted I7/I5 (blue) and I8/I5 (red) for varying overlap/D ratio (abscissa), using a step-function model (with intrafibrillar disorder) for SAXD intensity. Different line styles indicate varying levels of intrafibrillar disorder; dashed: $\mathrm{k}=0.00 \mathrm{~nm}^{2}$

(no disorder), solid: $\kappa=\kappa_{0}=1.75 \mathrm{~nm}^{2}$, dash-dot: $\kappa=\kappa_{1}=4.0 \mathrm{~nm}^{2}$. Circles indicate different stages $(a \rightarrow b \rightarrow c \rightarrow d)$ in the process, which correspond to the times in $C)$, where the labels $O(a-d)$ and $E(a-d)$ refer to the odd $\left(I_{7} / I_{5}\right)$ and even $\left(I_{8} / I_{5}\right)$ order ratio values at each stage respectively. An increase in disorder (vertical down arrow; $a \rightarrow b$ ) is followed by a change in O/D ratio (left-inclined arrow; $b \rightarrow c$ ), concluded by $a$ return to near-initial values $(c \rightarrow d)$. Arrows are displaced laterally in the $(a \rightarrow b)$ stage to avoid overlap. E) Schematics of the intrafibrillar-level mechanisms corresponding to the transient changes in D. Each vertical dark blue rod corresponds to a single tropocollagen molecule, which are aggregated to form a fibril. D-period, overlap (O) and gap (G) regions indicated on the leftmost schematic. As above, a, b, c and d correspond to points in Fig. 5D. Left to right: ordered arrangement of tropocollagen molecules with well-defined gap/overlap interface, followed by an increase in intrafibrillar disorder leading to a blurred interface, then a reduction in overlap zone and finally ordered intrafibrillar arrangement after recovery of pre-strain - for schematic 
clarity, the residual disorder at the last

$346 \times 285 \mathrm{~mm}(300 \times 300$ DPI $)$

1

2

3

4

5

6
7

8

9

10

11

12

13

14

15

16

17

18

19

20

21

22

23

24

25

26

27

28

29

30

31

32

33

34

35

36

37

38

39

40

41

42

43

44

45

46

47

48

49

50

51

52

53

54

55

56

57

58

59

60

ACS Paragon Plus Environment 


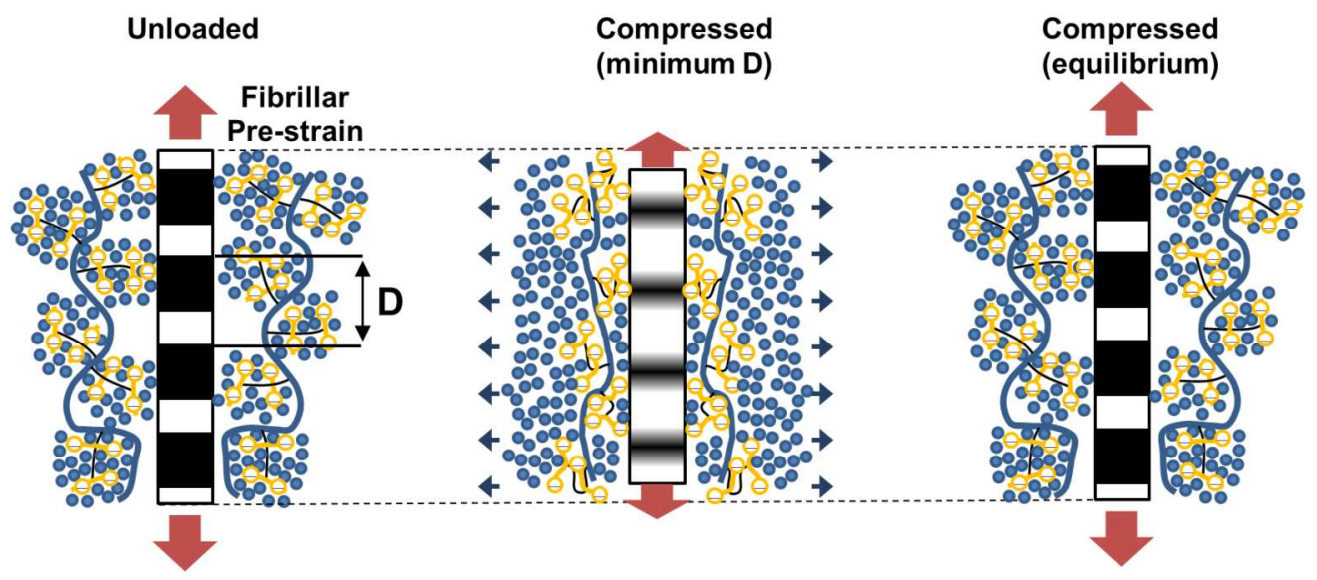

Figure 6. Fibrillar level mechanisms underlying transient change in pre-strain in cartilage: (A) Schematic of the cartilage ECM nanostructure at the fibrillar $(\sim 10-100 \mathrm{~nm})$ scale, with ordered Type II collagen fibrils (banded rods) surrounded by swollen, amorphous aggregates of negatively charged proteoglycans (orange circles) with a large number of loosely bound water molecules (blue circles)). Orange arrows indicated direction and relative magnitude of tensile pre-strain exerted by the proteoglycan aggregates on the collagen fibril. Left: Unloaded cartilage. Middle: Static compression of tissue is followed by a transient reduction of pre-strain in the collagen fibrils, possibly due to loss of water molecules and structural collapse in the proteoglycan network (blue arrows indicate direction of fluid flow). The reduction in pre-strain is shown by a decrease in D-period. In addition the gap/overlap interface in the collagen D-period banding pattern becoming increasingly blurred, suggesting an associated dis-ordering and loss of crystallinity in the collagen molecules. Right: As water molecules return to the proteoglycan aggregates there is a restoration of collagen fibril pre-strain and ordering/crystallinity. 


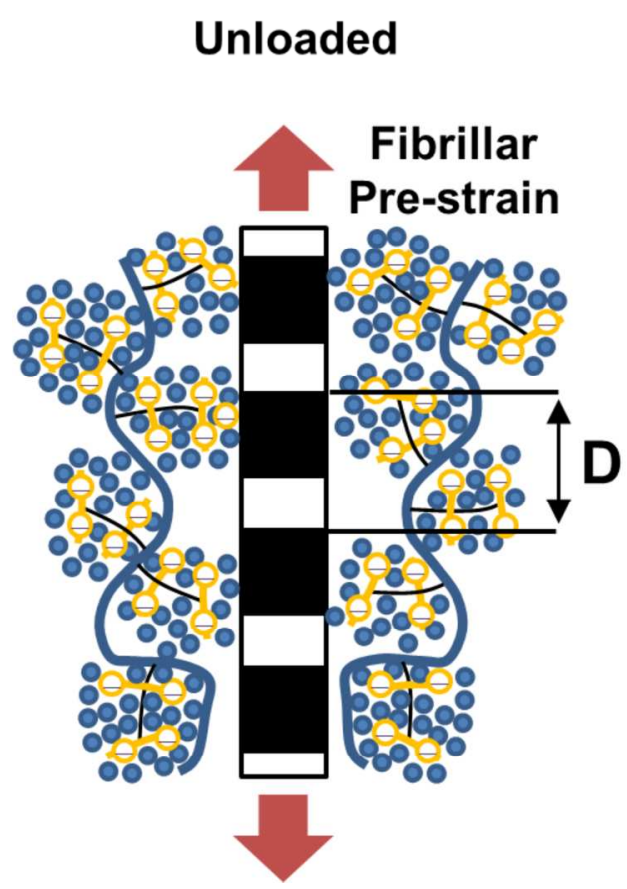

Compressed (minimum D)

Graphical abstract.

$184 \times 125 \mathrm{~mm}(300 \times 300$ DPI $)$ 